\title{
Improvement of the productivity of ecumicin, a novel anti-tuberculosis agent, from new Nonomuraea sp. MJM5123
}

\begin{abstract}
Ying-Yu Jin ${ }^{1,3}$, Jin-Yong Kim ${ }^{2,3}$, Seung Hwan Yang ${ }^{1}$, Hanki Lee ${ }^{1}$ and Joo-Won Suh ${ }^{1,2}$
Ecumicin is a novel anti-tuberculosis agent produced by Nonomuraea sp. MJM5123 as a new strain of actinomycetes. First, in order to increase the cell mass of Nonomuraea sp. MJM5123, we optimized the culture conditions with regard to carbon and nitrogen sources. The cell mass of Nonomuraea sp. MJM5123 increased by approximately twofold when glucose and soybean flour were used as carbon and nitrogen sources, respectively. For maximum production of ecumicin, we optimized the culture conditions by adding amino acids as building blocks for ecumicin, by adding vegetable oils and by controlling the temperature and $\mathrm{pH}$. Ecumicin production was two times higher with the addition of valine as the building blocks for ecumicin compared with the production in the absence of valine. Interestingly, with the addition of $1 \%$ corn oil, the production of ecumicin increased by 4.6-fold compared with the production in the absence of corn oil. Finally, by controlling the $\mathrm{pH}$ and temperature, we established an optimized culture condition in which Nonomuraea sp. MJM5123 produced $576 \mathrm{mg}$ ecumicin per litre of medium, which is about 50 times higher than in the control medium at $30^{\circ} \mathrm{C}$ and $\mathrm{pH} 7.0$.
\end{abstract}

The Journal of Antibiotics (2016) 69, 362-367; doi:10.1038/ja.2015.122; published online 9 December 2015

\section{INTRODUCTION}

Antibiotics have been used to control many diseases caused by pathogenic microorganism in human history and still occupy positions as the top drugs in related markets. ${ }^{1}$ Almost all antibiotics from actinomycetes are secondary metabolites that are in contrast to primary metabolites usually produced in stationary phase of actinomycetes growth. ${ }^{2}$ Production of antibiotics as secondary metabolites is tightly regulated by signals related to cellular differentiation. ${ }^{3}$ Hence, for maximum production of antibiotics, the optimization of various factors such as nutrients, other minerals, cultivation time, temperature and $\mathrm{pH}$ that affect the production of antibiotics by actinomycetes is an indispensable step. ${ }^{2}$ Moreover, the optimization of cultivation conditions for maximum production of antibiotics is also important in industrial strains because wild strains often produce antibiotics at a relatively low level. ${ }^{4}$

As previously reported, ecumicin is a novel anti-tuberculosis agent produced by Nonomuraea sp. MJM5123 as a new strain of actinomycetes. ${ }^{5,6}$ Ecumicin showed potent selective anti-tuberculosis activity against multidrug resistant as well as extensively drug resistant Mycobacterium tuberculosis and the $\mathrm{MIC}_{50}$ value was 4 and 50 times lower than for rifampicin and isoniazid, the first line drugs for the treatment of tuberculosis, respectively. ${ }^{6}$ Whole-genome sequencing of ecumicin resistant $M$. tuberculosis $\mathrm{H}_{37} \mathrm{Rv}$ and drug affinity responsive target stability have confirmed that ecumicin targets the $\mathrm{ClpCl}$ of M. tuberculosis directly. ${ }^{6}$ We determined that ecumicin can stimulate the ATPase activity of $\mathrm{ClpCl}$ and inhibit the proteolytic activity of the $\mathrm{ClpC} 1 / \mathrm{ClpP} / \mathrm{ClpP} 2$ complex that participates in protein homeostasis in M. tuberculosis. ${ }^{6}$ We also found out ecumicin is specifically accumulated in the cytoplasm of Nonomuraea sp. MJM5123 because the anti-tuberculosis activity of ecumicin was only observed in the methanol extract from the mycelium cake of this strain. ${ }^{6}$

In order to maximize the production of ecumicin as a lead compound for the treatment of tuberculosis, we demonstrate here how to increase the production of ecumicin by Nonomuraea sp. MJM5123 by controlling various factors such as the main nutrients, other minerals, vegetable oil, cultivation temperature and $\mathrm{pH}$.

\section{MATERIALS AND METHODS}

Isolation of Nonomuraea sp. MJM51234 from the soil sample The ecumicin-producing Nonomuraea sp. strain MJM5123 was isolated from a soil sample obtained in Hallasan National Park in Jeju Island, South Korea. The isolation method for soil actinomycetes was described in a previous report. ${ }^{7}$ The strain was maintained at the Extract Collection of Useful Microorganisms (ECUM) library at Myongji University, South Korea, and has been deposited at

${ }^{1}$ Center for Nurtaceutical and Pharmaceutical Materials, Yongin, Gyeonggi-do, Republic of Korea and ${ }^{2}$ Division of Biosciences and Bioinformatics, Myongii University, Yongin, Gyeonggi-do, Republic of Korea

${ }^{3}$ These authors contributed equally to this work.

Correspondence: Professor H Lee or Professor J-W Suh, Division of Biosciences and Bioinformatics, Myongji University, 116 Myongji-ro, Cheoin-gu, Yongin, Gyeonggi-do 17058, Republic of Korea.

E-mail: hklee@mju.ac.kr or jwsuh@mju.ac.kr

Received 7 May 2015; revised 25 October 2015; accepted 12 November 2015; published online 9 December 2015 
the Korean Collection for Type Cultures (KCTC) as a patent strain with the accession number KCTC 12178BP.

\section{Classification of Nonomuraea sp. MJM5123 by DNA molecular approaches}

Nonomuraea sp. MJM5123 genomic DNA was extracted following the recommended protocol with the CoreBio genomic DNA isolation Kit (South Korea). Its $16 \mathrm{~S}$ rDNA was amplified and sequenced using the universal primers: 27F (forward), 5'-AGAGTTTGATCATGGCTCAG-3'; and 1492R (reverse), 5'-AAGGAGGTGATCCARCCGCA-3'. The sequence was aligned with the closest Streptomyces strains in BLAST using CLUSTAL $\mathrm{X}^{8}$ and the phylogenic tree was constructed by the neighbor-joining method ${ }^{9}$ using MEGA4.0 software (Tempe, AZ, USA). ${ }^{10}$ The topology of the phylogenic tree was calculated by 1000 bootstrap replications. ${ }^{11}$ The genetic relatedness of Nonomuraea sp. MJM5123 to its most related strains was evaluated by the DNA-DNA slot-bolt hybridization method. ${ }^{12}$ The self-hybridization signal was taken as $100 \%$ and the relative intensities of genomic DNAs of other strains were determined as the percent similarity.

\section{Characterization of morphology of Nonomuraea sp. MJM5123 on} various agar plates

International Streptomyces Project (ISP) media were used to determine the color of aerial and substrate mycelia as well as the diffusible pigment. ${ }^{13}$ The morphological features of Nonomuraea sp. MJM5123 spores and its mycelia were observed by scanning electron microscopy (Hitachi, S-3500N, Tokyo, Japan). Temperature, $\mathrm{pH}$ and salinity conditions for Nonomuraea sp. MJM5123 growth was tested on ISP3 medium and the antibiotic susceptibility of Nonomuraea sp. MJM5123 was investigated on ISP2 medium for 2 weeks at $28^{\circ} \mathrm{C}$. Utilization of various sugars $(1 \%, \mathrm{w} / \mathrm{v})$ as the sole carbon source was evaluated on ISP9 medium.

\section{Maintenance of Nonomuraea sp. MJM5123}

Frozen vegetative mycelia of Nonomuraea sp. MJM5123 were used for the routine and reproducible preparation of ecumicin as follows. Nonomuraea sp. MJM5123 was inoculated into a $500 \mathrm{ml}$ baffled flask containing $70 \mathrm{ml}$ of activation medium (soluble starch $2.0 \%$, yeast extract $0.5 \%$, beef extract $0.3 \%$, tryptone $0.5 \%, \mathrm{CaCO}_{3} 0.2 \%, \mathrm{CoCl}_{2} \quad 0.0001 \%, \mathrm{MgSO}_{4} \cdot 7 \mathrm{H}_{2} \mathrm{O} 0.05 \%$ and NE-302 $0.05 \%$ at pH 7.2) and incubated on a rotary shaker at 200 r.p.m. for 3 days at $34^{\circ} \mathrm{C}$. The culture broth was thoroughly mixed with $50 \%$ glycerol at a 1:1 ratio $(\mathrm{v} / \mathrm{v})$, and $5 \mathrm{ml}$ aliquots were stored at $-80^{\circ} \mathrm{C}$ until required.
Cultivation of Nonomuraea sp. MJM5123 in $500 \mathrm{ml}$ flask

For flask cultivation, first, a 5-ml frozen vegetative mycelia aliquot was transferred to a $500-\mathrm{ml}$ baffled flask containing $50 \mathrm{ml}$ activation medium and cultured at a shaking speed of 200 r.p.m. for $54 \mathrm{~h}$ at $34^{\circ} \mathrm{C}$ to activate Nonomuraea sp. MJM5123. And then, a 5-ml aliquot of the seed culture broth was inoculated in a $500-\mathrm{ml}$ baffled flask containing $50 \mathrm{ml}$ modified medium and cultivated with 200 r.p.m. of shaking for $60 \mathrm{~h}$ at $34^{\circ} \mathrm{C}$.

\section{Monitoring cell growth of Nonomuraea sp. MJM5123 by carbon} and nitrogen source

In order to select a carbon source for maximum cell growth of Nonomuraea sp. MJM5123, the microorganism was cultivated in basal medium (1\% of carbon source, $0.1 \%$ of nitrogen source, $0.05 \%$ of $\mathrm{NaCl}, 0.05 \%$ of $\mathrm{MgSO}_{4} \cdot 7 \mathrm{H} 2 \mathrm{O}, 0.05 \%$ of $\mathrm{K}_{2} \mathrm{HPO}_{4}, 0.1 \%$ of $\mathrm{CaCO}_{3}, 0.001 \%$ of $\mathrm{FeSO}_{4} \cdot 7 \mathrm{H}_{2} \mathrm{O}$ and $\mathrm{pH}$ 7.1) by adding six carbon sources individually. After cultivation, the cell growth of Nonomuraea sp. MJM5123 was monitored by measuring the packed mycelium volume (PMV) as follows. $10 \mathrm{ml}$ of the culture broth of Nonomuraea sp. MJM5123 was sampled at various times and centrifuged at $450 \mathrm{~g}$ for $10 \mathrm{~min}$. After selecting the carbon source, the nitrogen source was selected using the same method.

\section{Analysis of ecumicin contents produced by Nonomuraea sp. MJM5123}

Mycelium of Nonomuraea sp. MJM5123 was collected by centrifugation of the $10 \mathrm{ml}$ culture broth at $1900 \mathrm{~g}$ for $20 \mathrm{~min}$ at room temperature. To harvest the extract containing ecumicin from the mycelium, $20 \mathrm{ml}$ of methanol was added to the mycelium and the mixture was shaken vigorously with an agitator for $30 \mathrm{~min}$ at room temperature. The sample was prepared by filtering the methanol extract through a $0.2 \mu \mathrm{m}$ syringe filter. To monitor ecumicin, $10 \mu \mathrm{l}$ of the filtrate was analyzed by Waters HPLC System with YMC-Pack ODS-A column $(5 \mu \mathrm{m}, 4.6 \times 250 \mathrm{~mm}$, Kyoto, Japan) with $40 \%$ aqueous acetonitrile solution buffered in $20 \mathrm{~mm}$ sodium phosphate $(\mathrm{pH} 8.0)$ as the mobile phase. The flow rate was maintained at $1.2 \mathrm{ml} \mathrm{min}^{-1}$ and ecumicin was detected by $\mathrm{UV}$ absorbance at $280 \mathrm{~nm}$ for analysis. For comparison of the productivity of ecumicin depending on the change of nutrients, Glucose soluble starch (GSS) medium was used as basal medium.

Effect of amino acids as ecumicin building blocks and vegetable oils in ecumicin production by Nonomuraea sp. MJM5123

To discover the effect of amino acids as ecumicin building blocks, Nonomuraea sp. MJM5123 was cultivated in modified medium with all nitrogen sources completely removed to avoid unexpected supplementation as amino acids by a

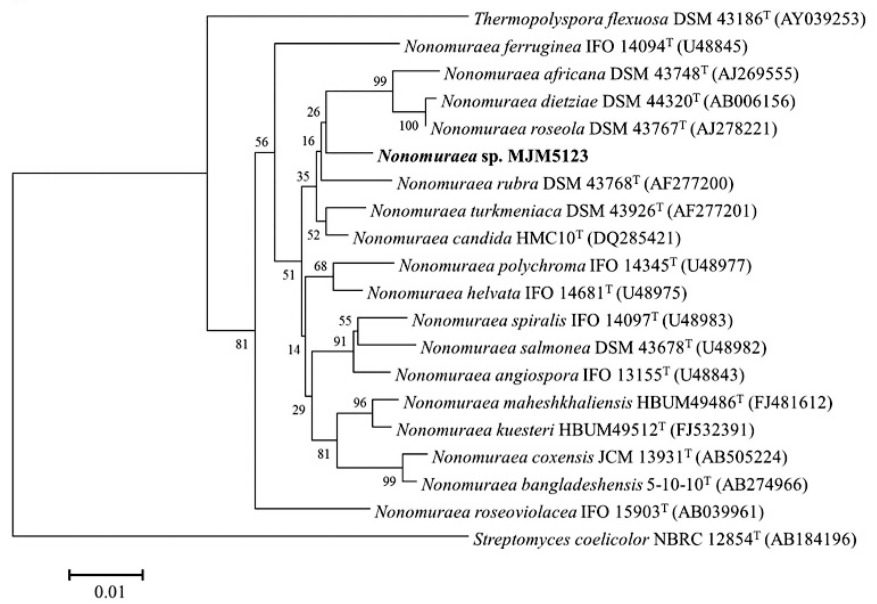

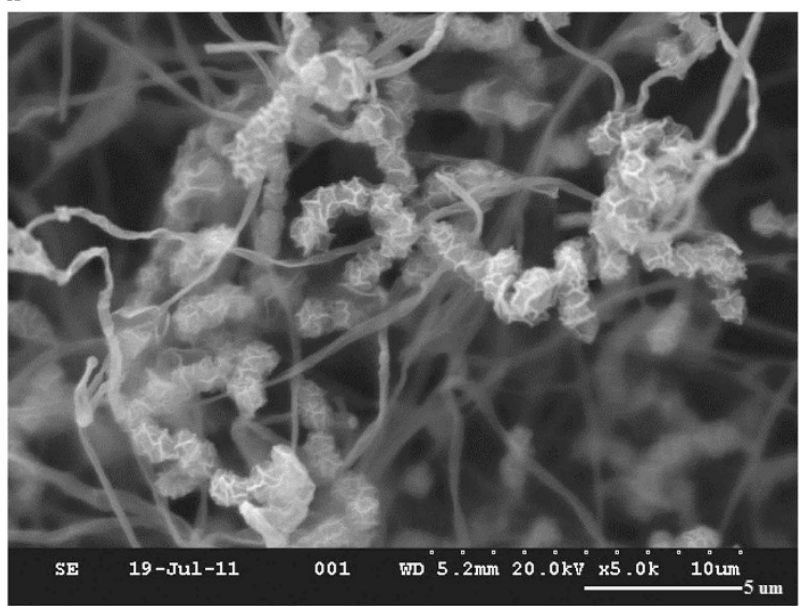

Figure 1 Characteristics of Nonomuraea sp. MJM5123. (a) Phylogenetic analysis of Nonomuraea sp. MJM5123 based on 16S rDNA. 16S rDNA of Nonomuraea sp. MJM5123 was analyzed with Nonomuraea type strains and other actinomycetes. (b) Spore and mycelium of Nonomuraea sp. MJM5123 by scanning electron microscopy. Scale bar indicated $5 \mu \mathrm{m}$. 
the degradation of other nitrogen sources. All amino acids for this experiment were added separately at $0.05 \%(\mathrm{w} / \mathrm{v})$ in minimal medium. The effect of vegetable oils was monitored as follows. Nonomuraea sp. MJM5123 was cultivated in modified medium through the addition of soybean, corn, olive, canola and grape-seed oil separately.

\section{Cultivation of Nonomuraea sp. MJM5123 in fermenter scale}

The main fermentation was carried out in a 7L fermenter (KoBiotech, South Korea) with 51 working volume. A total of $500 \mathrm{ml}$ seed culture broth was transferred to 4.51 of modified medium. The culture was maintained at an agitation speed of 600 r.p.m. and 0.3 v.v.m. of aeration for $144 \mathrm{~h}$ at $34^{\circ} \mathrm{C}$.

\section{Effect of cultivation temperature and $\mathrm{pH}$ on ecumicin production} by Nonomuraea sp. MJM5123 in fermenter scale

After seeding Nonomuraea sp. MJM5124, the temperature was maintained at 28,30 or $34{ }^{\circ} \mathrm{C}$ till the completion of cultivation. The culture broth was sampled at the appropriate temperature to monitor ecumicin production. In order to monitor ecumicin production by $\mathrm{pH}$ control, the culture medium was maintained at $\mathrm{pH} 6.0$ and 7.0 by the addition of $\mathrm{H}_{2} \mathrm{SO}_{4}$ at $30^{\circ} \mathrm{C}$.

\section{RESULTS}

Phylogenetic relatedness based on the 16S rRNA gene of Nonomuraea sp. MJM5123 indicated that the strain belonged to the genus Nonomuraea but was not strongly clustered with other isolates. MJM5123 was found to be similar to Nonomuraea rubra DSM 43768T (98.2\% 16S rRNA gene similarity), Nonomuraea roseola DSM 43767T (98.0\% 16 S rRNA gene similarity) and Nonomuraea dietziae DSM 44320T (97.5\% 16S rRNA gene similarity; Figure 1a). Genome-wide comparision using DNA-DNA hybridization analysis between Nonomuraea sp. MJM5123 and the above three strains resulted in hybridization value below $70 \%$, which are consistent with Nonomuraea sp. MJM5123 representing a novel species of the genus Nonomuraea (Table 1). The spores of Nonomuraea sp. MJM5123 were

Table 1 Genetic relatedness of Nonomuraea sp. MJM5123 with closely related strains by DNA-DNA hybridization

\begin{tabular}{lc}
\hline Taxa & Nonomuraea sp. MIM5123 (\%) \\
\hline Nomonuraea Sp. MJM5123 & 100 \\
N. dietziae DSM 44320 & 65.8 \\
N. roseola DSM 43767 & 65.5 \\
N. rubra DSM 43768 & 34.8
\end{tabular}

The self-hybridization signal of Nonomuraea sp. MJM5123 genomic DNA was $100 \%$ and relative signals with other strains genomic DNA were evaluated as the percent similarity. Tindicates type strain.

Table 2 Phenotypic features of Nonomuraea sp. MJM5123 in ISP media

\section{MJM5123}

ISP medium

Growth/reverse color/aerial mycelium/sporulation

\begin{tabular}{ll}
\hline ISP2 & $++/$ beige/ $-/-$ \\
ISP3 & $+/$ beige/++/++ \\
ISP4 & $+/$ beige/ $/$ - \\
ISP5 & $+/$ beige/ $/$ - \\
ISP6 & $+/$ beige/ $-/-$ \\
ISP7 & $+/$ beige/ $-/-$
\end{tabular}

Abbreviation: ISP, International Streptomyces Project.

Growth and sporulation on aerial mycelium were scored as follows: ++, good; +, moderate - , no growth and no spores formed. open spirals with a rugose surface (Figure 1b). Filamentous aerial and substrate mycelium were beige in color on all media tested, and the mycelium was not fragmented. Aerial mycelia and spores were formed only on ISP3 agar medium (Table 2). Table 3 shows the physiological and biochemical characteristics of the MJM5123 strain, which did not produce any diffusible pigments or melanin. The MJM5123 strain grew in a temperature range of $20-40^{\circ} \mathrm{C}$ and a $\mathrm{pH}$ range of 5.0-9.0 and tolerated up to $3 \% \mathrm{NaCl}$ (Table 3). The strain utilized diverse sugars, hexoses, pentose, alcohol sugars and disaccharides as the sole carbon sources and was susceptible to apramycin, kanamycin, vancomycin and thiostrepton but resistant to ampicillin (Table 3 ).

We discovered that ecumicin is mainly accumulated inside the cells of Nonomuraea sp. MJM5123, ${ }^{6}$ so we first focused on increasing the cell mass of this strain by carbon and nitrogen sources before increasing the yield of ecumicin. In the presence of $1 \%$ glucose, the cell mass of Nonomuraea sp. MJM5123 was measured to be 3.9\% and this amount was higher than with any other carbon sources in this experiment (Figure 2a). For nitrogen sources, the addition of $0.1 \%$ of cotton seed meal, dry yeast and $\left(\mathrm{NH}_{4}\right)_{2} \mathrm{SO}_{4}$ effectively increased the cell mass of the strain compared with 15 other nitrogen sources (Figure 2b). So, hereafter, cultivation of Nonomuraea sp. MJM5123 was carried out in $1 \%$ glucose and $0.1 \%$ of cotton seed meal, dry yeast and $\left(\mathrm{NH}_{4}\right)_{2} \mathrm{SO}_{4}$.

As previously reported, production of bioactive secondary metabolites by actinomycetes was stimulated by adding vegetable oils during cultivation. ${ }^{14-16}$ So, we tested whether the productivity of ecumicin was increased by adding vegetable oils. Compared with the results in the absence of the four vegetable oils tested, the productivity of ecumicin was higher in all groups in which vegetable oils were supplemented.

Table 3 Physiological and biochemical properties of Nonomuraea sp. MJM5123

\begin{tabular}{|c|c|c|c|}
\hline Characteristics $^{a}$ & Description & Characteristics $^{b}$ & Description \\
\hline \multirow{3}{*}{$\begin{array}{l}\text { Utilization of sole carbon } \\
\text { source: }\end{array}$} & & Cell wall amino acids: & \\
\hline & & & \\
\hline & & Glycine & + \\
\hline Glucose & ++ & L-diaminopimelic acid & + \\
\hline Sucrose & ++ & D-diaminopimelic acid & + \\
\hline Fructose & + & & \\
\hline Mannitol & ++ & $\begin{array}{l}\text { Whole-cell saccharide } \\
\text { analysis: }\end{array}$ & \\
\hline Xylose & ++ & & \\
\hline Arabinose & ++ & Glucose & + \\
\hline Rhamnose & ++ & Galactose & + \\
\hline Raffinose & \pm & Mannose & + \\
\hline Inositol & \pm & Arabinose & ND \\
\hline Galactose & ++ & Ribose & ND \\
\hline Maltose & ++ & Xylose & + \\
\hline Mannose & ++ & Rhamnose & ND \\
\hline Melanin pigment & & $\begin{array}{l}\text { Antibiotic resistance } \\
\left(50 \mu \mathrm{g} \mathrm{ml}^{-1}\right)\end{array}$ & \\
\hline With tyrosine & - & & \\
\hline \multirow[t]{2}{*}{ Without tyrosine } & - & Apramycin & - \\
\hline & & Kanamycin & - \\
\hline $\mathrm{pH}$ range for growth & $5.0-9.0$ & Vancomycin & - \\
\hline Growth temperature & $20-40^{\circ} \mathrm{C}$ & Thiostrepton & - \\
\hline Growth salinity & Up to $3 \%$ & Ampicillin & + \\
\hline
\end{tabular}

${ }^{a}$ Characteristics were scored as: ++ , positive; + , moderate; \pm , poor; - , negative. ${ }^{b}$ Characteristics were scored as: + , detected; ND, non-detected. 
a

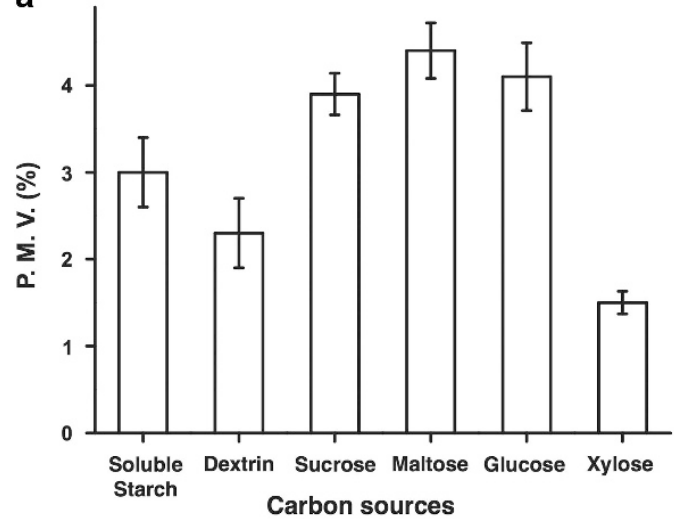

b

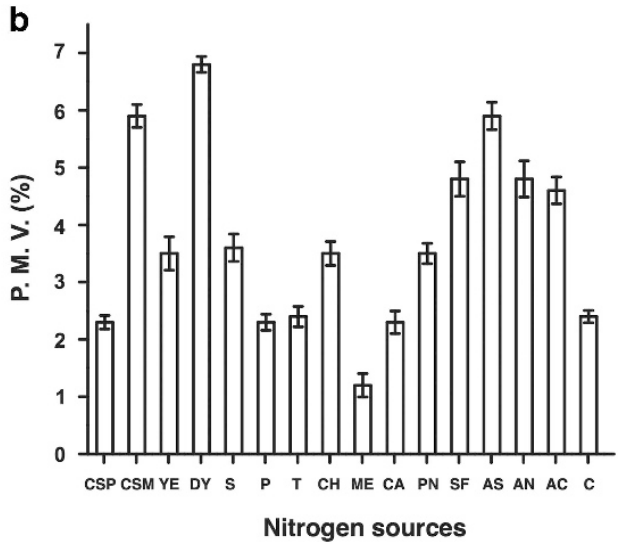

Figure 2 Cell mass of Nonomuraea sp. MJM51234 by addition of carbon and nitrogen sources. (a) By carbon sources (b) By nitrogen sources. AC, ammonium chloride, AN, ammonium nitrate; AS, ammonium sulfate; C, control as Glucose soluble starch (GSS) medium; CH, casein hydrolysate; CSM, corn seed meal; CSP, corn steep powder; DY, dried yeast; P, peptone; PN, potassium nitrate; S, soytone; SF, soybean flour; T, tryptone.
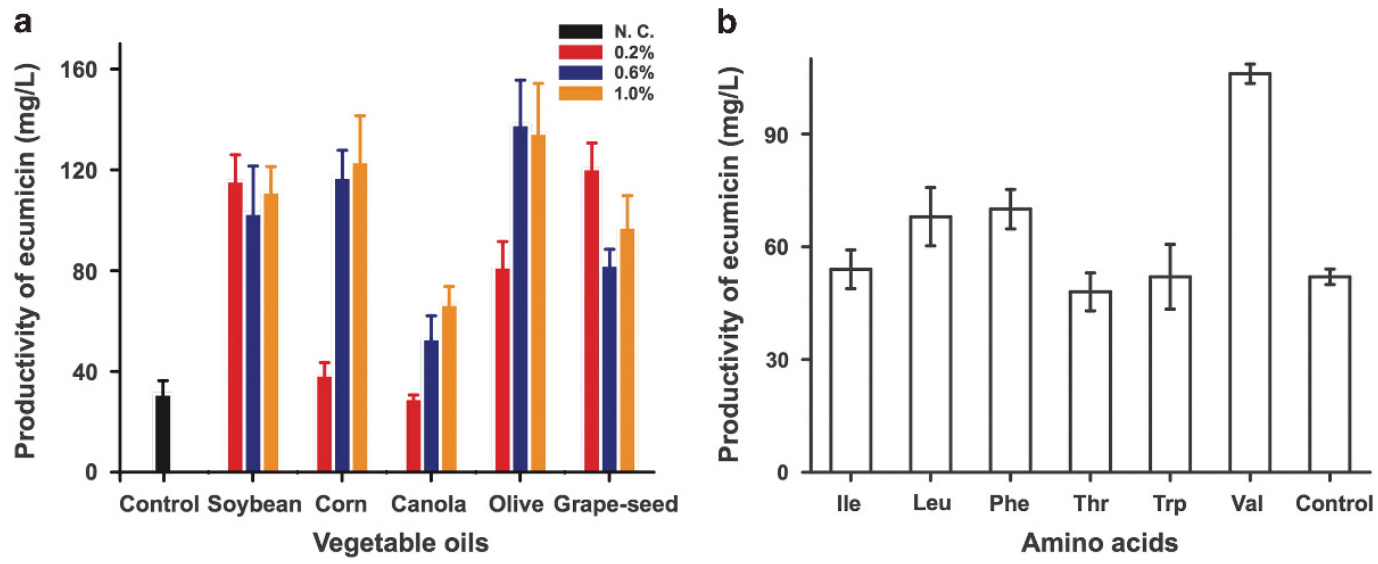

Figure 3 Ecumicin production by Nonomuraea sp. MJM5123 in addition of vegetable oils and amino acids. Ecumicin productivity was measured by analytical HPLC by $\mathrm{C}_{18}$ column. (a) By addition of vegetable oils (b) By addition of amino acids.
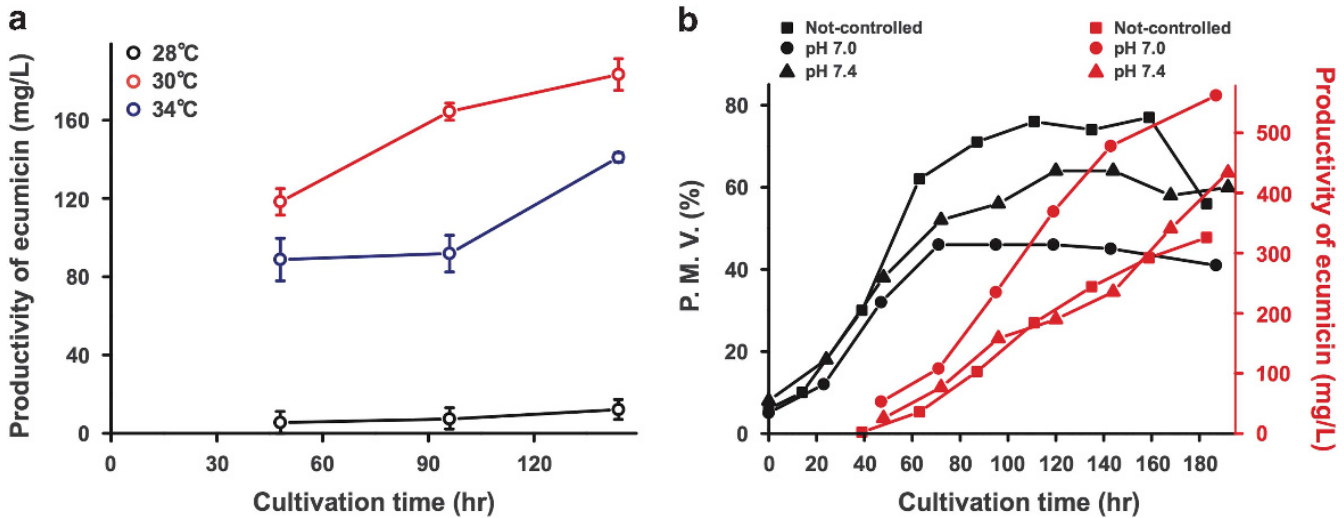

Figure 4 Ecumicin production corresponding to growth temperatures and medium $\mathrm{pH}$ in $7 \mathrm{~L}$ fermenter jar. Ecumicin productivity was measured by analytical HPLC by $\mathrm{C}_{18}$ column. (a) By growth temperatures. (b) By medium $\mathrm{pH}$; left and right axis indicate packed mycelium volume (PMV) and production of ecumicin, respectively.

Also, notably, in the presence of 0.6 and $1.0 \%$ olive oil, the productivity of ecumicin increased fourfold, compared with the negative control (Figure 3a). As mentioned above, ecumicin was found to be cyclic depsipeptide synthesizing by NRPS gene cluster of Nonomuraea sp. MJM5123. ${ }^{6}$ So, we investigated how the addition of various amino acids to the culture medium could alter the ecumicin production. With the addition of $0.05 \%$ valine, the productivity was twofold higher than the productivity with seven other amino acids (Figure $3 \mathrm{~b}$ ). In addition, in all groups with supplement amino acids, ecumicin was produced at higher amounts compared with the negative control. 
Table 4 The final optimized medium condition for maximum production of ecumcin

\begin{tabular}{|c|c|}
\hline Material & Composition (\%) \\
\hline Glucose & 1 \\
\hline Cotton seed meal & 0.1 \\
\hline Dry yeast & 0.1 \\
\hline$\left(\mathrm{NH}_{4}\right)_{2} \mathrm{SO}_{4}$ & 0.1 \\
\hline $\mathrm{K}_{2} \mathrm{HPO}_{4}$ & 0.1 \\
\hline Olive oil & 1 \\
\hline L-valine & 0.05 \\
\hline $\mathrm{CaCl}_{2}^{\mathrm{a}}$ & 0.01 \\
\hline $\mathrm{MgSO}_{4} \cdot 7 \mathrm{H}_{2} \mathrm{O}^{\mathrm{a}}$ & 0.08 \\
\hline $\mathrm{FeSO}_{4} \cdot 7 \mathrm{H}_{2} \mathrm{O}^{\mathrm{a}}$ & 0.001 \\
\hline $\mathrm{pH}$ control & 7.0 by the addition of $\mathrm{H}_{2} \mathrm{SO}_{4}$ \\
\hline
\end{tabular}

andicates minerals added for this fermentation. ${ }^{17}$

Based on the results of the flask test, we carried out a scale-up experiment in a $7 \mathrm{~L}$ fermenter. First, we monitored the $\mathrm{pH}$ of the culture broth and the PMV after controlling the temperature because temperature can affect the growth of Nonomuraea sp. MJM5123 and consequently, the production of ecuminin. The $\mathrm{pH}$ at the end of cultivation was found to be similar and was measured at around 8.0 at each temperature while the PMV value was different at each temperature.

In particular, when production of ecumicin began at $48 \mathrm{~h}$, the PMV at $28^{\circ} \mathrm{C}$ was lower than for the other temperatures. At the end of the culture, the PMV at $34^{\circ} \mathrm{C}$ continued to increase. This increase may be disadvantageous in terms of the production of secondary metabolites by actinomycetes. However, the PMV at $30^{\circ} \mathrm{C}$ was good at $48 \mathrm{~h}$ and was maintained at the end of cultivation, unlike the PMV at $28^{\circ} \mathrm{C}$ and $34^{\circ} \mathrm{C}$. The PMV data was also correlated with the production of ecumicin in the $7 \mathrm{~L}$ fermenter experiment (Figure $4 \mathrm{a}$ ). Production of ecumicin began after $48 \mathrm{~h}$ at a rate of $120 \mathrm{mgl}^{-1}$ and increased to $180 \mathrm{mgl}^{-1}$ by the end of cultivation. During cultivation at 28 and $30^{\circ} \mathrm{C}$, ecumicin production was $10 \mathrm{mgl}^{-1}$ and $140 \mathrm{mgl}^{-1}$, respectively (Figure 4a). Therefore, we determined that the optimal temperature for producing ecumicin is $30^{\circ} \mathrm{C}$ through this experiment.

Finally, to examine whether $\mathrm{pH}$ control during cultivation affects the production of ecumicin or not, we monitored the production of ecumicin and the cell mass with $\mathrm{pH}$ control by adding $\mathrm{H}_{2} \mathrm{SO}_{4}$ during fermentation. After fermentation, the amount of ecumicin was found to be $576 \mathrm{mgl}^{-1}$ and $420 \mathrm{mgl}^{-1}$ in $\mathrm{pH} 7.0$ and $\mathrm{pH} 7.4$, respectively (Figure $4 \mathrm{~b}$ ). In the condition without $\mathrm{pH}$ control, ecumicin was $318 \mathrm{mgl}^{-1}$ (Figure 4b). Interestingly, the PMV of Nonomuraea sp. MJM5123 was found to be different from the production of ecumicin. At the end of fermentation, the PMV was higher at $\mathrm{pH} 7.4$ and rather low without $\mathrm{pH}$, suggesting the secondary metabolic pathway for synthesizing ecumcin is delicately regulated by controlling $\mathrm{pH}$ and the increase of cell mass of Nonomuraea sp. MJM5123 as a primary metabolic pathway is not directly linked to the production of ecumicin.

\section{DISCUSSION}

Nonomuraea sp. MJM5123, which is isolated from soil, is a rare strain of actinomycetes identified as a new strain of the Nonomuraea species. Generally, rare actinomycetes often produce unexpected and unidentified metabolites, compared with known actinomycetes. Therefore, the isolation of rare actinomycetes is more advantageous in terms of finding new metabolites. In this regard, we found that
Nonomuraea sp. MJM5123 produces ecumicin, which has potent anti-tuberculosis activity and is a macrocyclic tridecapeptide composed of 13 amino acids, through screening anti-tuberculosis activity using whole-cell Mycobacterium tuberculosis.

Before optimizing the culture condition to maximize the production of ecumicin, we observed that Nonomuraea sp. MJM5123 produced $10 \mathrm{mgl}^{-1}$ of ecumicin in GSS medium, which is usually used for the isolation and cultivation of actinomycetes (data not shown). This amount of ecumicin was considered very small as wild-type actinomycetes often produce low amounts of antibiotics. In this study, we tried to optimize ecumicin production through the composition of nutrients, control of temperature and $\mathrm{pH}$. First, the increase of cell mass was an important factor for maximizing ecumicin production because ecumicin mainly accumulates in the cells of Nonomuraea sp. MJM5123. Use of glucose and soybean flour as the respective carbon and nitrogen sources resulted in the highest dry cell weight, so we selected glucose and soybean flour for the main fermentation since the use of cheap materials is favorable in terms of pilot scaled production. Second, we measured the production of ecumicin corresponding to the addition of vegetable oils and amino acids as ecumicin building blocks. Addition of vegetable oils increased the production of ecumicin above the control and in particular, addition of olive oil ( 0.6 and $1.0 \%)$ increased the production of ecumicin by threefold. Improvement of ecumicin production by adding vegetable oils corresponds to the results of previous research in regards to the effect of vegetable oil on antibiotic production by actinomycetes. Generally, the addition of vegetable oils not only increases the total growth in the presence of carbon sources but also provides easily consumable sources of carbon and energy in the absence of carbon sources. Therefore, extended energy supply by the addition of vegetable oils can increase the production of antibiotics like ecumicin even if energy was no longer supplied after complete consumption of carbon sources. Third, we monitored ecumicin production by adding various amino acids because ecumicin is a macrocyclic tridecapeptide composed of 13 amino acids. Interestingly, with the addition of valine, the production of ecumicin increased twofold compared with the negative control. This result indicates that valine was used as the main building block, with seven valines in ecumicin during the biosynthesis process. Valine is also used in the protein precursors for the biosynthesis of primary metabolites.

Finally, we monitored how ecumicin was produced by Nonomuraea sp. MJM5123 by controlling the temperature and $\mathrm{pH}$ in a $7 \mathrm{~L}$ fermenter. Briefly, maximum production of ecumicin was accomplished during cultivation at $30^{\circ} \mathrm{C}$ under $\mathrm{pH}$ 7.0. In this condition, production of ecumicin was measured at $576 \mathrm{mgl}^{-1}$, which corresponds to a 57.6-fold increase compared with the production of ecumicin in GSS medium (Table 4). Generally, $\mathrm{pH}$ and temperature are essential factors in the production of secondary metabolites by actinomycetes as well as for the primary metabolism of these bacteria. Currently, we have isolated and analyzed the ecumicin biosynthetic gene cluster in Nonomuraea sp. MJM5123 through whole-genome sequencing (data not shown) and are attempting to enhance the production of ecumicin by metabolic engineering based on modification of the ecumicin biosynthetic gene cluster.

\section{CONFLICT OF INTEREST}

The authors declare no conflict of interest. 


\section{ACKNOWLEDGEMENTS}

This work was supported by the Cooperative Research Program for Agriculture Science and Technology Development (Project No. PJ01128901 and Project No. PJ01133001), Rural Development Administration, Republic of Korea. Author contributions: HL and J-WS designed research as co-corresponding author.; Y-YJ and J-YK optimized the culture condition for maximum productivity and analyzed the productivity of ecumicin equally. SHY supported the the maintenance of Nonomuraea sp. MJM5123 and carried out the phylogenetic study using $16 \mathrm{~s}$ rDNA. HL and J-WS wrote this manuscript. All authors discussed the results and commented on this manuscript.

1 Saleem, M. et al. Antimicrobial natural products: an update on future antibiotic drug candidates. Nat. Prod. Rep. 27, 238-254 (2010).

2 Rokem, J. S., Lantz, A. E. \& Nielsen, J. Systems biology of antibiotic production by microorganisms. Nat. Prod. Rep. 24, 1262-1287 (2007).

3 Zhu, H., Sandiford, S. K. \& van Wezel, G. P. Triggers and cues that activate antibiotic production by actinomycetes. J. Ind. Microbiol. Biotechnol. 41, 371-386 (2014).

4 Straathof, A. J., Panke, S. \& Schmid, A. The production of fine chemicals by biotransformations. Curr. Opin. Biotechnol. 13, 548-556 (2002).

5 Gao, W. et al. Discovery and characterization of the tuberculosis drug lead ecumicin. Organ. Lett. 16, 6044-6047 (2014).

$6 \mathrm{Gao}, \mathrm{W}$. et al. The cyclic peptide ecumicin targeting $\mathrm{ClpC} 1$ is active against Mycobacterium tuberculosis in vivo. Antimicrob. Agents Chemother. 59, 880-889 (2015).
7 Kim, W. G., Yoon, T. M., Kwon, H. J. \& Suh, J. W. Talosins A and B: new isoflavonol glycosides with potent antifungal activity from Kitasatospora kifunensis MJM341. II. Physicochemical properties and structure determination. J. Antibiot. (Tokyo) 59 640-645 (2006).

8 Thompson, J. D., Gibson, T. J., Plewniak, F., Jeanmougin, F. \& Higgins, D. G. The CLUSTAL $X$ windows interface: flexible strategies for multiple sequence alignment aided by quality analysis tools. Nucleic Acids Res. 25, 4876-4882 (1997).

9 Saitou, N. \& Nei, M. The neighbor-joining method: a new method for reconstructing phylogenetic trees. Mol. Biol. Evol. 4, 406-425 (1987).

10 Tamura, K., Dudley, J., Nei, M. \& Kumar, S. MEGA4: Molecular Evolutionary Genetics Analysis (MEGA) software version 4.0. Mol. Biol. Evol. 24, 1596-1599 (2007).

11 Efron, B., Halloran, E. \& Holmes, S. Bootstrap confidence levels for phylogenetic trees. Proc. Natl Acad. Sci. USA 93, 13429-13434 (1996).

12 Kafatos, F. C., Jones, C. W. \& Efstratiadis, A. Determination of nucleic acid sequence homologies and relative concentrations by a dot hybridization procedure. Nucleic Acids Res. 7, 1541-1552 (1979).

13 Shirling, E. B. \& Gottlieb, D. Methods for characterization og Streptomyces species. Int. J. Syst. Evol. Microbiol. 16, 313-340 (1966).

14 Jones, A. M. \& Porter, M. A. Vegetable oils in fermentation: beneticial effects of low-level supplementation. J. Ind. Microbiol. Biotechnol. 21, 203-207 (1998).

15 Hamedi, J., Malekzadeh, F. \& Niknam, V. Improved production of erythromycin by Saccharopolyspora erythraea by various plant oils. Biotechnol. Lett. 24, 697-700 (2002).

16 Maranesi, G. L., Baptista-Neto, A., Hokka, C. O. \& Badino, A. C. Utilization of vegetable oil in the production of clavulanic acid by Streptomyces clavuligerus ATCC 27064. World Microb. Biotechnol. 21, 509-514 (2005).

17 Calam, C. T. Process Development in Antibiotic Fermentations (Cambridge University Press, New York, NY, USA, 2008). 